\title{
A new massive double-lined spectroscopic binary system: The Wolf-Rayet star WR 68a ${ }^{\star}$ (Research Note)
}

\author{
A. Collado ${ }^{1,2}$, R. Gamen ${ }^{3}$, R. H. Barbá ${ }^{4}$, and N. Morrell ${ }^{5}$ \\ ${ }^{1}$ Instituto de Ciencias Astronómicas, de la Tierra y del Espacio (ICATE), CONICET, Avda. España 1512 Sur, J5402DSP, San Juan, \\ Argentina \\ e-mail: acollado@icate-conicet.gob.ar \\ 2 Facultad de Ciencias Exactas, Físicas y Naturales, Universidad Nacional de San Juan, San Juan, Argentina \\ ${ }^{3}$ Instituto de Astrofísica de La Plata, CONICET, Facultad de Ciencias Astronómicas y Geofísicas, Universidad Nacional de La Plata, \\ Paseo del Bosque s/n, B1900FWA, La Plata, Argentina \\ ${ }^{4}$ Departamento de Física y Astronomía, Universidad de La Serena, Av. Juan Cisternas 1200 Norte, La Serena, Chile \\ 5 Las Campanas Observatory, Carnegie Observatories, Casilla 601, La Serena, Chile
}

Received 26 August 2014 / Accepted 30 July 2015

\section{ABSTRACT}

\begin{abstract}
Double-lined spectroscopic binary systems, containing a Wolf-Rayet and a massive O-type star, are key objects for the study of massive star evolution because these kinds of systems allow the determination of fundamental astrophysical parameters of their components. We have performed spectroscopic observations of the star WR 68a as part of a dedicated monitoring program of WR stars to discover new binary systems. We identified spectral lines of the two components of the system and disentangled the spectra. We measured the radial velocities in the separated spectra and determined the orbital solution. We discovered that WR 68a is a doublelined spectroscopic binary with an orbital period of 5.2207 days, very small or null eccentricity, and inclination ranging between 75 and 85 deg. We classified the binary components as WN6 and O5.5-6. The WN star is less massive than the O-type star with minimum masses of $15 \pm 5 M_{\odot}$ and $30 \pm 4 M_{\odot}$, respectively. The equivalent width of the He II $\lambda 4686$ emission line shows variations with the orbital phase, presenting a minimum when the WN star is in front of the system. The light curve constructed from available photometric data presents minima in both conjunctions of the system.
\end{abstract}

Key words. binaries: spectroscopic - stars: Wolf-Rayet - stars: individual: WR 68a - stars: fundamental parameters

\section{Introduction}

Observational works dedicated to the study of multiplicity among massive stars, e.g. Barbá et al. (2010), Sana et al. (2013), and many references there in, provide evidence that the fraction of $\mathrm{O}+\mathrm{OB}$ systems is high, greater than $50 \%$. This situation is not completely replicated by the available data for Wolf-Rayet (WR) stars. There are about 50 binary systems detected among the galactic WR stars (less than 10\% of the known whole sample), and only 24 of them are known double-lined spectroscopic systems (SB2). These systems are key objects because they allow the determination of some fundamental astrophysical parameters for their component stars. Moreover, double-lined eclipsing systems allow the determination of stellar masses and radii in a direct and reliable way, thus providing strong constraints on stellar physics and evolutionary models. In this context, the discovery of these kinds of systems is highly relevant.

In an attempt to search for new binary systems among faint WR stars, a spectroscopic monitoring of southern galactic WR stars is underway. This survey was started in 2007 and its main results published to date are the discovery of the SB2 WR 62a, one of the most conspicuous radial-velocity

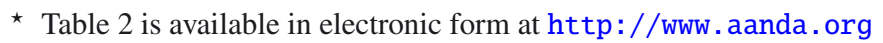

(RV) variable stars in our sample (Collado et al. 2013, hereafter Paper I), and WR 35a (Gamen et al. 2014).

WR 68a (SMSNPL13, $v=14.41$ ) was identified as a WR star by Shara et al. (1999). No other data or analysis on this star were found in the bibliography, thus it was included in our survey.

In this paper, we present the first spectroscopic and photometric analysis of WR 68a, demonstrating that this is an SB2 system. The paper is organised as follows: in Sect. 2, we describe the observations and data reduction. In Sect. 3, we present the spectral analysis of the system's components, the measurement and analysis of radial velocities, and the study of the available photometry. In Sect. 4, we summarise our results.

\section{Observations and data reduction}

We obtained thirty-six spectroscopic observations of WR 68a between 2007 and 2013. The observations were carried out with the 2.15-m J. Sahade telescope at the Complejo Astronómico El Leoncito (CASLEO, Argentina), the 4-m V. Blanco telescope at Cerro Tololo Inter-American Observatory (CTIO, Chile), and the 2.5-m du Pont and the 6.5-m Magellan Baade telescopes at Las Campanas Observatory (LCO, Chile). See Table 1 for a summary of the observations. Immediately after or before the stellar 
Table 1. Details of the observing material.

\begin{tabular}{|c|c|c|c|c|c|c|c|c|c|}
\hline $\begin{array}{c}\text { Date-obs. } \\
\text { UT }\end{array}$ & $n$ & $\begin{array}{c}\text { Sp. coverage } \\
{[\AA]}\end{array}$ & Observat. & $\begin{array}{c}\text { Telesc. } \\
{[\mathrm{m}]}\end{array}$ & Spectr. $^{a}$ & $\begin{array}{l}\text { Grating } \\
{\left[1 \mathrm{~mm}^{-1}\right]}\end{array}$ & Detector & $\begin{array}{c}\text { Dispersion } \\
{\left[\AA \mathrm{pix}^{-1}\right]}\end{array}$ & $\begin{array}{c}\text { Resolving }^{b} \\
\text { power }\end{array}$ \\
\hline 2007, Mar. 30 & 2 & $3650-6700$ & CTIO & 4 & $\mathrm{R}-\mathrm{C}$ & 632 & Loral 3k & 1.01 & 1300 \\
\hline 2007, Apr. $1-3$ & 4 & $3650-6700$ & CTIO & 4 & $\mathrm{R}-\mathrm{C}$ & 632 & Loral 3k & 1.01 & 1300 \\
\hline 2008, Apr. 19-23 & 7 & $3650-6700$ & CTIO & 4 & $\mathrm{R}-\mathrm{C}$ & 632 & Loral 3k & 1.01 & 1300 \\
\hline 2008, Jun. 16 & 1 & $3650-6750$ & LCO & 6.5 & IMACS f/4 & 600 & SITe (Mosaic 1) & 0.4 & 3300 \\
\hline 2009, Mar. 24-27 & 7 & $4030-5590$ & CASLEO & 2.15 & REOSC SD & 600 & Tek1024 & 1.63 & 1000 \\
\hline 2009, Jul. 23 & 1 & $3890-5520$ & LCO & 2.5 & $\mathrm{~B}-\mathrm{C}$ & 1200 & Marconi\#1 & 0.79 & 2500 \\
\hline 2009, Jul. 26 & 1 & $3540-6700$ & LCO & 2.5 & $\mathrm{~B}-\mathrm{C}$ & 600 & Marconi\#1 & 1.55 & 1200 \\
\hline 2009, Jul. 27 & 1 & $3890-5520$ & LCO & 2.5 & $\mathrm{~B}-\mathrm{C}$ & 1200 & Marconi\#1 & 0.79 & 2500 \\
\hline 2009, Aug. 14-17 & 3 & $4040-5700$ & CASLEO & 2.15 & REOSC SD & 600 & Tek1024 & 1.63 & 1000 \\
\hline 2010 , Apr. $10-11$ & 2 & $3930-5600$ & CASLEO & 2.15 & REOSC SD & 600 & Tek1024 & 1.63 & 1000 \\
\hline 2010, Aug. 2-8 & 4 & $3930-5600$ & CASLEO & 2.15 & REOSC SD & 600 & Tek1024 & 1.63 & 1000 \\
\hline 2011, Apr. 6-11 & 2 & $3930-5600$ & CASLEO & 2.15 & REOSC SD & 600 & Tek1024 & 1.63 & 1000 \\
\hline 2013, May 12 & 1 & $3930-5600$ & CASLEO & 2.15 & REOSC SD & 600 & Tek1024 & 1.63 & 1000 \\
\hline
\end{tabular}

Notes. " $n$ ": number of spectra taken per run. ${ }^{(a)}$ Details of the spectrographs can be found in the user manuals of the respective observatories. (b) The spectral resolving power $(R=\lambda / \Delta \lambda)$ were measured using $\Delta \lambda$ as the FWHM of calibration lamp emission lines.

integration, at the same telescope position, we obtained comparison lamp spectra. We reduced the spectra using $\operatorname{IRAF}^{1}$ standard procedures.

\section{Results and discussion}

\subsection{The spectrum of WR 68 a}

The spectrum of WR 68a shows noticeable emission lines of helium, carbon, and nitrogen ions, and also several absorption lines of hydrogen and helium superimposed onto the emissions. As we prove below, these absorption lines move in anti-phase with respect to the emissions, indicating that WR 68a is a double-lined WR + OB binary system.

To separate the individual components in the spectrum of WR 68a, we applied a disentangling method similar to that developed by González \& Levato (2006). Briefly, the method consists of shifting all the spectra to the common RV for one component and co-adding them so that the features of the other component are diluted. In a second step, the pure spectrum of one component (template) is subtracted to obtain a new RV determination. These steps are repeated in an iterative way. The method was only applied to the CTIO and LCO spectra because CASLEO data have lower signal-to-noise ratio $(\mathrm{S} / \mathrm{N})$, and thus they introduce noise into the mean spectra of each component of the binary.

The individual spectra (see Fig. 1) were used to perform a more detailed analysis of both stars. Relative intensities of N III, NIV, He II, and C IV emission lines in the spectra indicate a WN6o spectral type (according to the criteria given by Smith et al. 1996) ${ }^{2}$, in good agreement with Shara et al. (1999).

Absorption lines of $\mathrm{H}(\mathrm{H} \alpha, \mathrm{H} \beta, \mathrm{H} \gamma, \mathrm{H} \delta)$, He I $(\lambda \lambda 4026$, 4471, and 5875), He II ( $\lambda \lambda 4542,4686$, and 5411), and C III $\lambda 5696$ emission were identified in the disentangled spectrum, indicating the presence of an O-type star. To determine its spectral type, we used the MGB code (Maíz Apellániz et al. 2012), which facilitates a visual comparison with standard O-type spectra (Sota et al. 2011, 2014). Thus, we classified the

\footnotetext{
1 IRAF is distributed by the National Optical Astronomy Observatories, which are operated by the Association of Universities for Research in Astronomy, Inc., under cooperative agreement with the National Science Foundation.

2 The "o" means that no hydrogen is observed in the spectrum.
}

O-type star as O5.5-6. Because of poor $\mathrm{S} / \mathrm{N}$ in the blue region of the spectrum and probable residuals of the wings of the $\mathrm{H} \delta$ and He II $\lambda 4686$ emission introduced by the disentangling method, we could not derive a reliable luminosity class for the secondary spectrum.

\subsection{Measurement and analysis of radial velocities}

The radial velocities ( $\mathrm{RVs}$ ) were determined by means of crosscorrelation (using the IRAF FXCOR task) between the separated spectra of each component and the corresponding template. For each emission line, an appropriate spectral range was used to determine the RV thereof. In the case of the RVs of the absorption lines, we determined a mean RV using the spectral ranges of $\mathrm{H} \beta, \mathrm{H} \gamma$, and $\mathrm{H} \delta$. The individual RVs of the measured emission lines according to the heliocentric Julian dates (HJD) of the observations, including N IV $\lambda 4058$, the blend N III $\lambda \lambda 4634-40-42$, He II $\lambda \lambda 4686$ and 5411, and C IV $\lambda 5812$ emissions, and the mean $\mathrm{RV}$ of $\mathrm{H} \delta, \mathrm{H} \gamma, \mathrm{H} \beta$ absorption lines are listed in Table 2.

We searched for periodicities in the RVs of the He II $\lambda 4686$ emission line, using the method developed by Marraco \& Muzzio (1980). This algorithm computes, for each trial period, the variances from the best-fitting straight line in each phase interval. The most probable period obtained is $5.22 \mathrm{~d}$. Then, by means of the GBART ${ }^{3}$ code, we individually fit orbital solutions to the RVs for the emission and absorption lines. As all lines show a similar periodicity, we adopted the straight mean of the periods obtained from each data set, i.e. $P=5.2207 \pm 0.0005 \mathrm{~d}$, as the binary period. Thus, with the fixed period, we independently determined the orbital parameters for the emission and the mean of the absorption lines, and we show these in Table 3. The orbital solutions for each emission line are illustrated in Fig. 2.

As expected, each data set presents different systemic velocities, semi-amplitudes, times of periastron passage, and maximum RV. For example, the blend N III $\lambda \lambda 4634-40-42$ presents the lowest semi-amplitude and the C IV $\lambda 5812$ emission appears to have the largest. This is a common behaviour in $\mathrm{WN}+\mathrm{OB}$ systems, generally related to the fact that emission lines are formed

\footnotetext{
3 GBART is an improved version of the program for the determination of the orbital elements for spectroscopic binaries originally written by Bertiau \& Grobben (1968), developed by F. Bareilles, and available at http://www . iar . unlp. edu . ar/ fede/pub/gbart
} 
A. Collado et al.: A new massive binary system: The Wolf-Rayet star WR 68a $(R N)$

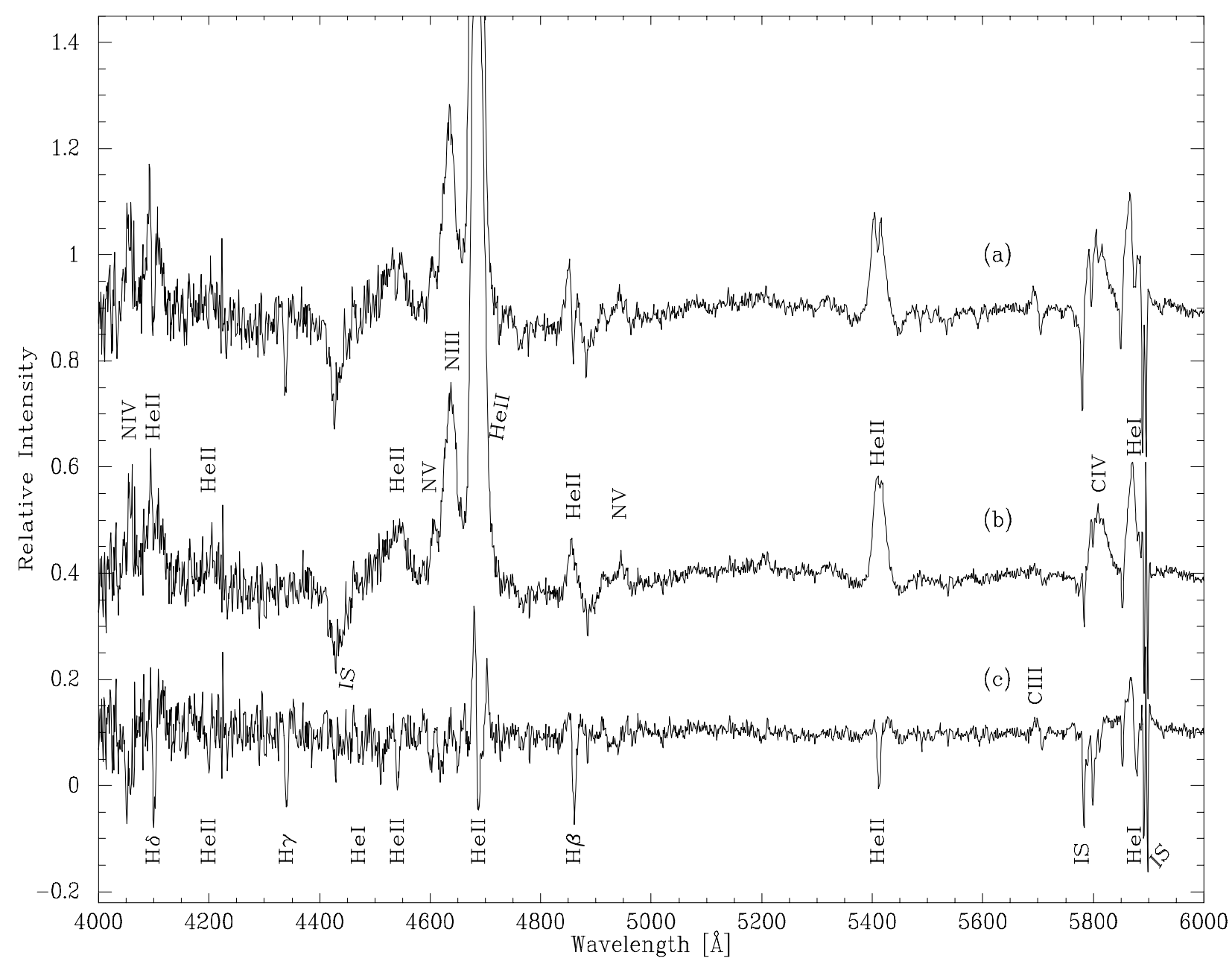

Fig. 1. (a) Continuum rectified spectrum of WR 68a before disentangling; (b) the WR component after removing the O-type; (c) the remaining spectrum of the O-type star. The spectra are vertically shifted for comparison.

Table 3. Orbital solutions corresponding to the radial velocities of the N IV, N III, He II, and C IV emission lines, and mean abs.

\begin{tabular}{ccccccc}
\hline \hline Parameter & N IV & N III & He II & He II & C IV & Mean abs. \\
& 4057.76 & 4640.64 & 4685.68 & 5411.52 & 5811.98 & $\mathrm{H} \delta$ - $\mathrm{H} \gamma$-H $\beta$ \\
\hline$P$ [days $]$ & & & $5.2207 \pm 0.0005$ \\
$V_{0}\left[\mathrm{~km} \mathrm{~s}^{-1}\right]$ & $-159 \pm 7$ & $-245 \pm 5$ & $4 \pm 3$ & $22 \pm 5$ & $-373 \pm 4$ & $-146 \pm 4$ \\
$K\left[\mathrm{~km} \mathrm{~s}^{-1}\right]$ & $314 \pm 11$ & $307 \pm 7$ & $331 \pm 4$ & $331 \pm 7$ & $352 \pm 7$ & $158 \pm 6$ \\
$e$ & $0.05 \pm 0.03$ & $0.08 \pm 0.02$ & $0.05 \pm 0.01$ & $0.04 \pm 0.02$ & $0.06 \pm 0.01$ & $0.20 \pm 0.04$ \\
$w$ [degrees $]$ & $350 \pm 37$ & $206 \pm 17$ & $111 \pm 12$ & $134 \pm 30$ & $157 \pm 19$ & $262 \pm 9$ \\
$T_{\text {Periast }}[\mathrm{d}]^{*}$ & $5036.9 \pm 0.5$ & $5039.7 \pm 0.2$ & $5038.4 \pm 0.2$ & $5038.8 \pm 0.4$ & $5039.4 \pm 0.3$ & $5038.4 \pm 0.1$ \\
$T_{\text {RVmax }}[]^{*}$ & $5037.1 \pm 0.5$ & $5036.7 \pm 0.2$ & $5036.8 \pm 0.2$ & $5036.9 \pm 0.4$ & $5037.2 \pm 0.3$ & $5039.4 \pm 0.1$ \\
$T_{\text {WRinfront }}[\mathrm{d}]^{*}$ & $5038.3 \pm 0.5$ & $5038.1 \pm 0.2$ & $5038.1 \pm 0.2$ & $5038.2 \pm 0.4$ & $5038.5 \pm 0.3$ & $5035.9 \pm 0.1$ \\
$a$ sin i $\left[R_{\odot}\right]$ & $32 \pm 1$ & $32 \pm 1$ & $34 \pm 1$ & $34 \pm 1$ & $36 \pm 1$ & $16 \pm 1$ \\
$\sigma\left[\mathrm{km} \mathrm{s}^{-1}\right]$ & 31.49 & 35.57 & 23.51 & 37.37 & 17.12 & 21 \\
\hline
\end{tabular}

Notes. ${ }^{(*)}$ HJD-2 455000.

in asymmetric (not barycentric) regions at different depths in the expanding envelope (see e.g. Niemela \& Moffat 1982; Niemela et al. 1995).

It is also noteworthy that the RVs of the absorption lines are anti-phased with respect to the emission lines, thus indicating that they belong to the O-type component of the system. The semi-amplitude of its orbit is lower than that of the WN (regardless of which emission line is used), which means that the O-type star is the more massive star in the system.

Eccentricities other than zero are not common in binary systems containing classical He-burning WN components. Only
WR 97 is known to have a significant $e(0.1 \pm 0.04$, Gamen 2004). We checked the robustness of the found $e$ with a test of significance given by Lucy \& Sweeney (1971) and it resulted to be spurious in all of the emission lines (they do not reach the 5\% level of significance for accepting eccentric orbits), but are significant in the absorption lines. This feature should be interpreted with tailored models appropriate to this context of close binaries, i.e. heating hemispheres by a hot companion, asymmetric emission-line forming regions, colliding wind zone, etc. On the other hand, as is shown below, the light curve presents two dips at 0.5 phase intervals, also pointing to a circular orbit. In the 


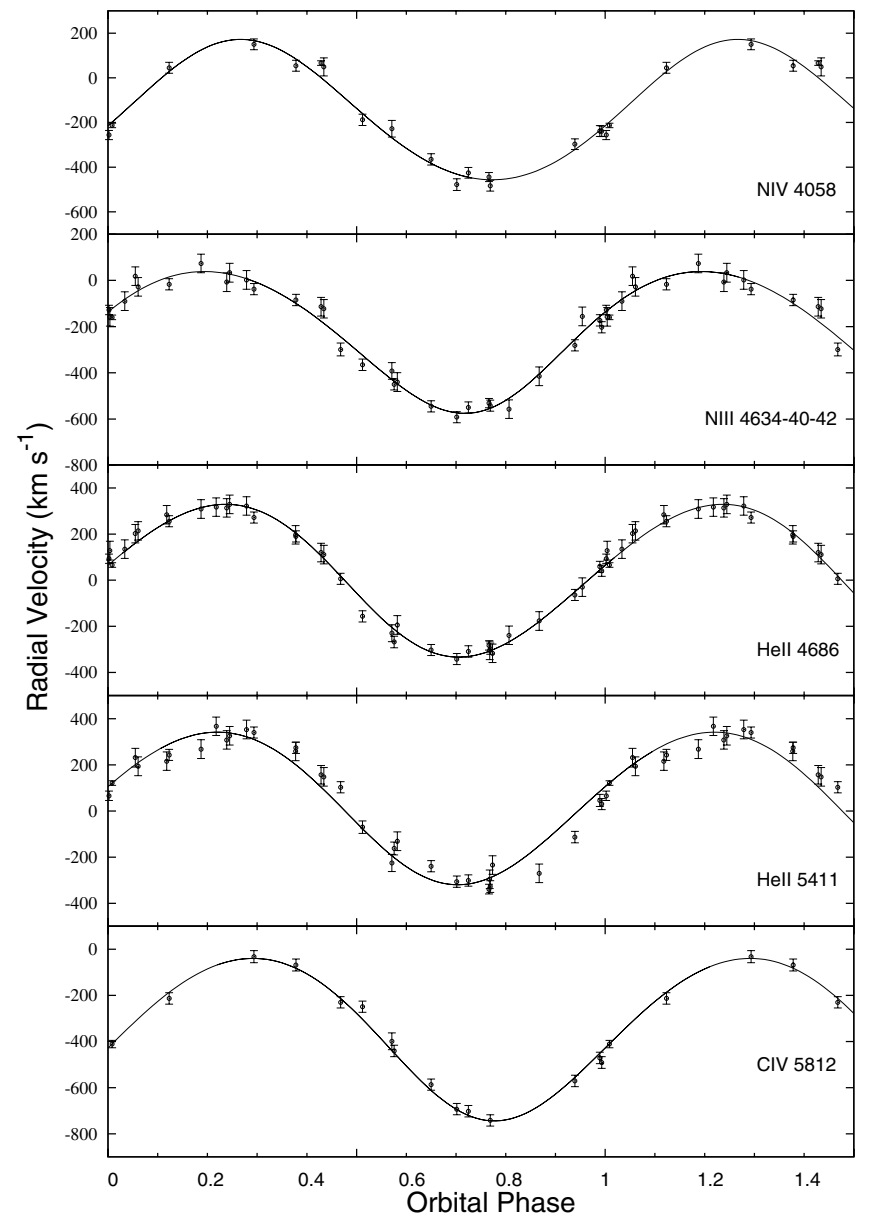

Fig. 2. Radial velocities of N IV, N III, He II, and C IV emission lines. Continuous curves represent the orbital solutions in Table 3.

following analysis, a circular orbit is assumed for the WR 68a binary system.

To determine the orbital solution of both components simultaneously, we assumed that the orbital motion of the WN star is better represented by the mean RVs of the nitrogen emission lines, and the motion of the O-type star is better depicted by the most conspicuous lines in the O-type spectrum. We shifted the RVs of emission lines by the difference between the barycentric velocities obtained for the individual solutions to match the lines of the O-type star component. We performed an iterative process to determine the orbital solution, rejecting those points whose $\mathrm{O}-\mathrm{C}$ values were three times larger than the rms of the whole solution in each trial. The orbital parameters and the RV orbits are shown in Table 4 and Fig. 3, respectively.

The minimum mass derived for the O-type star is very similar to the theoretical value for an $\mathrm{O} 5.5-6 \mathrm{~V}$ star, as calibrated by Martins et al. (2005), 31-33 $\mathrm{M}_{\odot}$. Therefore, the orbital inclination should be close to $90 \mathrm{deg}$, and we can expect to observe photometric variability (eclipses, for instance).

\subsection{Analysis of the emission-line profiles and available photometry}

We analysed the behaviour of the equivalent width (EW) and full width at half maximum (FWHM) of some lines at different orbital phases. We found that the EW and FWHM of the He II $\lambda \lambda 4686,5411$ and N IV $\lambda 4058$ emission lines present some variability modulated by the orbital period, which is very
Table 4. Cicular orbital elements of WR 68a using the mean of emission lines of nitrogen for the WN component and the mean of $\mathrm{H} \beta, \mathrm{H} \gamma$, and $\mathrm{H} \delta$ absorption lines for the O-type component.

\begin{tabular}{cc}
\hline \hline$P[\mathrm{~d}]$ & 5.2207 (fixed) \\
$K_{\mathrm{WR}}$ & $295 \pm 7$ \\
$K_{\mathrm{O}}\left[\mathrm{km} \mathrm{s}^{-1}\right]$ & $144 \pm 9$ \\
$V_{0}\left[\mathrm{~km} \mathrm{~s}^{-1}\right]$ & $-145 \pm 4$ \\
$T_{\mathrm{RVmax}}[\mathrm{d}]^{*}$ & $5039.56 \pm 0.02$ \\
$T_{\mathrm{WRinfront}}[\mathrm{d}]^{*}$ & $5040.86 \pm 0.02$ \\
$a_{\mathrm{WR}} \sin i\left[R_{\odot}\right]$ & $33 \pm 1$ \\
$a_{\mathrm{O}} \sin i\left[R_{\odot}\right]$ & $15 \pm 1$ \\
$M_{\mathrm{WR}} \sin ^{3} i\left[M_{\odot}\right]$ & $15 \pm 5$ \\
$M_{\mathrm{O}} \sin ^{3} i\left[M_{\odot}\right]$ & $30 \pm 4$ \\
$q_{\mathrm{WR} / \mathrm{O}}$ & $0.49 \pm 0.04$ \\
$\sigma\left[\mathrm{km} \mathrm{s}^{-1}\right]$ & 32.12 \\
\hline
\end{tabular}

Notes. ${ }^{(*)}$ HJD-2 455000 .

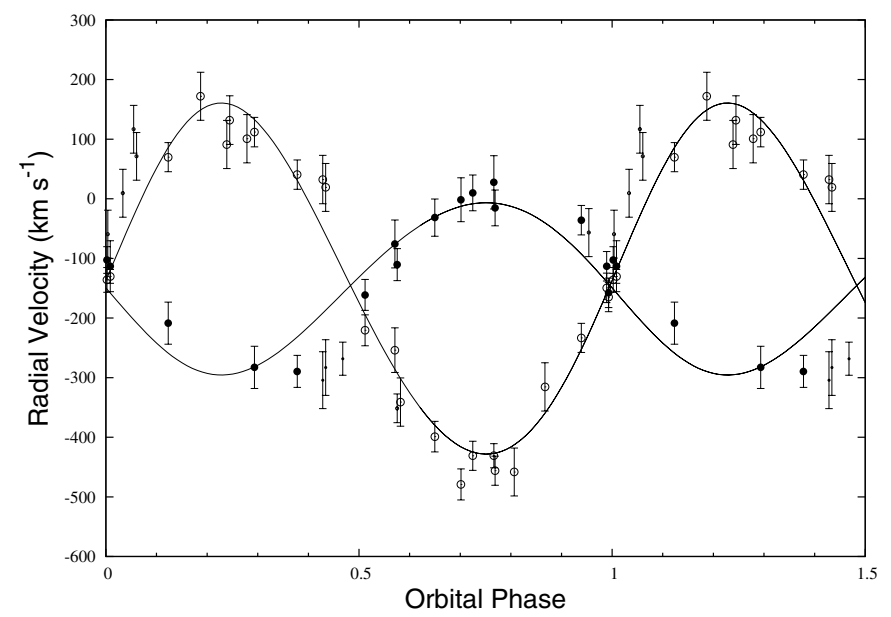

Fig. 3. WR 68a RV orbit represented by the mean of emission lines of nitrogen for the WN component (open circles) and the mean of $\mathrm{H} \beta, \mathrm{H} \gamma$, and $\mathrm{H} \delta$ absorption lines for the secondary component (close circles). Points not considered in the solution are plotted with smaller sizes.

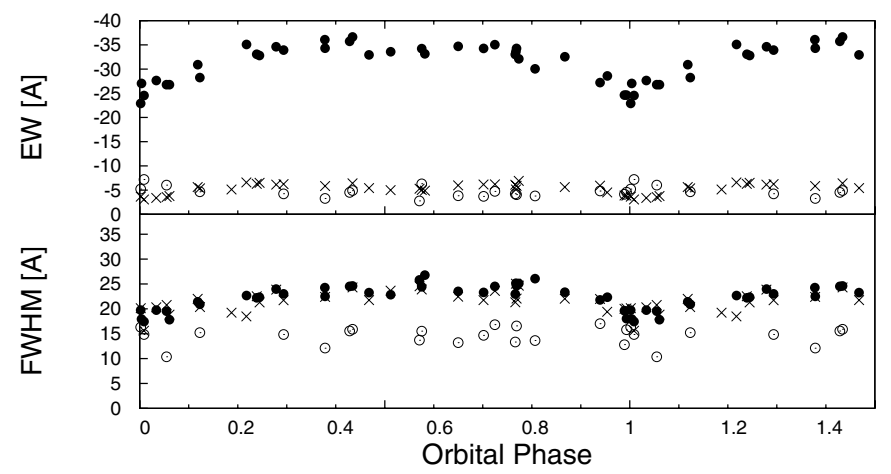

Fig. 4. EW and FWHM, as a function of orbital phase, measured in the He II $\lambda 4686$ (close circles), N IV $\lambda 4058$ (open circles), and He II $\lambda 5411$ (crosses) emission lines.

noticeable in the He II $\lambda 4686$ line (see Fig. 4). This line becomes fainter around phase $\phi=0.0$, i.e. when the WN star is in the front of the system. A similar effect has previously been observed in WR 62a (see Paper I) and in the intriguing LBV HD 5980 (Foellmi et al. 2008), and it has been explained as due to the emission line having an additional non-stellar component, which originates in the colliding wind region.

We also analysed the data collected by the All Sky Automated Survey (ASAS; Pojmański 2001). We constructed 


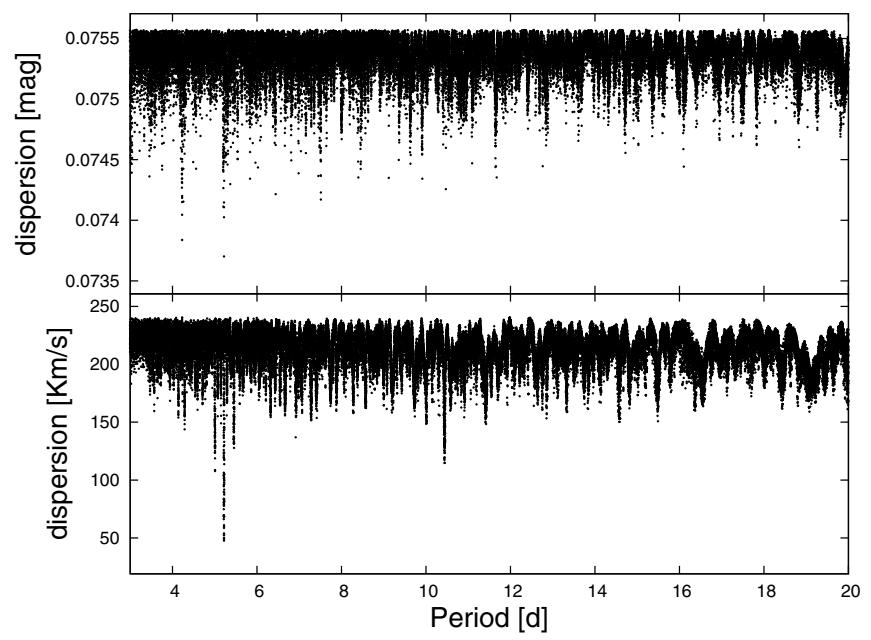

Fig. 5. Periodograms obtained for the ASAS data (top) and for the He II $\lambda 4686$ emission line (bottom).

the light curve (see Fig. 6, where we also show the averaged points for each bin of $\phi=0.05$ ), and noted two dips: one in phase 0.0 (when the WN-type component passes in front of the system) and the other, less pronounced, in phase 0.5 . To prove it is not fortuitous, the ASAS data were searched for periodicities, applying the same algorithm used for the RVs. The most likely period found is $P=5.22 \mathrm{~d}$, which is similar to the spectroscopic period (both periodograms are compared in Fig. 5).

The similarity between the light curves of WR 62a and WR 68a is notorious, however, the dip at $\phi=0.0$ in the light curve of WR 68a is shallower and wider than that observed in WR 62a. The resemblance of these light curves to that of V444 Cyg, a WN5+O6 binary system, is also remarkable (see Fig. 2 of Eriş \& Ekmekçi 2011). The photometric data of the ASAS survey are not suitable for a detailed analysis because of the faintness of WR 68a. Notwithstanding that, we adjusted a simple Wilson-Devinney (WD) model (Wilson \& Devinney 1971) by means of the PHOEBE code (Prša \& Zwitter 2005), to the RV of both stars and the photometric data together. We adopted the theoretical stellar mass and radii for an $\mathrm{O} 5.5-6 \mathrm{~V}$ star from Martins et al. (2005) and adjusted the stellar parameters of the WN6 component. We found that the light curve can be reasonably fitted with an orbital inclination ranging between 75 and $85 \mathrm{deg}$. These values imply for the WN component a radius between 4.5 and $7.4 R_{\odot}$, comparable with those estimated for the WN5 component of V444 Cyg (Eriş \& Ekmekçi 2011, and references therein).

\section{Conclusions}

We have discovered that WR 68a is a double-lined binary system composed of a WN6 star and an O 5.5-6 type component in a orbital period of $5.2207 \mathrm{~d}$. We found that the O-type star is more massive than the WN component with minimum masses of $30 \pm 4 M_{\odot}$ and $15 \pm 5 M_{\odot}$ for the O-type and WN stars, respectively.

We also detected a minimum in the EW of the He II $\lambda 4686$ emission when the WN star is in front of the system, likely due to the eclipse of an additional component of this line originated in the colliding winds region. From the analysis of the available photometric data (ASAS), we found a variability, i.e. two minima in the light curve during both

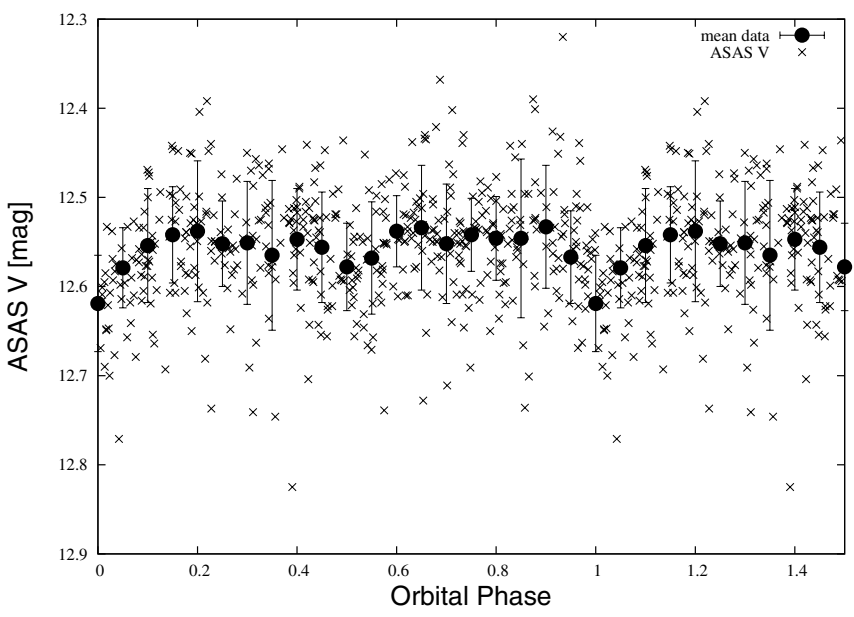

Fig. 6. ASAS $V$-band photometry of WR 68a. Crosses depict the ASAS data, and the circles, the averaged in phase bins of 0.05 with their respective standard deviation.

conjunctions of the system, which we interpreted as eclipses.

WR 68a becomes in an important astronomical target, as accurate multi-band photometric and higher resolution spectroscopic follow-up observations will allow to determine the stellar parameters, as radius and masses, for both massive components. WR $68 \mathrm{a}$ is thus a target of choice to obtain direct properties of stars in a WR+O system, hence, to obtain valuable constraints to confront evolutionary models of massive stars.

Acknowledgements. We are grateful to the referee for valuable comments, corrections, and suggestions, which helped improve the paper. We thank the directors and staff of CASLEO, LCO, and CTIO for support and hospitality during our observing runs. CASLEO is operated under agreement between the Consejo Nacional de Investigaciones Científicas y Técnicas de la República Argentina and the National Universities of La Plata, Córdoba and San Juan. RHB acknowledges support from FONDECYT Regular Project No. 1140076.

\section{References}

Barbá, R. H., Gamen, R., Arias, J. I., et al. 2010, in Rev. Mex. Astron. Astrofis., 38,30

Bertiau, F., \& Grobben, J. 1968, Ric. Astr. Spec. Vat., 8, 1

Collado, A., Gamen, R., \& Barbá, R. H. 2013, A\&A, 552, A22

Eriş, F. Z., \& Ekmekçi, F. 2011, Astron. Nachr., 332, 616

Foellmi, C., Koenigsberger, G., Georgiev, L., et al. 2008, Rev. Mex. Astron. Astrofis. 44, 3

Gamen, R. 2004, Ph.D. Thesis, La Plata University

Gamen, R., Collado, A., Barbá, R., Chené, A.-N., \& St-Louis, N. 2014, A\&A, 562, A 13

González, J. F., \& Levato, H. 2006, A\&A, 448, 283

Lucy, L. B., \& Sweeney, M. A. 1971, AJ, 76, 544

Maíz Apellániz, J., Pellerin, A., Barbá, R. H., et al. 2012, 465, 484

Marraco, H., \& Muzzio, J. 1980, PASP, 92, 700

Martins, F., Schaerer, D., \& Hillier, D. J. 2005, A\&A, 436, 1049

Niemela, V. S., \& Moffat, A. 1982, Ap.J., 259, 213

Niemela, V. S., Cabanne, M. L., \& Bassino, L. P. 1995, Rev. Mex. Astron. Astrofís., 31, 45

Pojmański, G. 2001, in Small Telescope Astronomy on Global Scales, eds. B. Paczynski, W.-P. Chen, \& C. Lemme, IAU Colloq. 183, ASP Conf. Ser., 246,53

Prša, A., \& Zwitter, T. 2005, ApJ, 628, 426

Sana, H., de Koter, A., de Mink, S. E., et al. 2013, A\&A, 550, A107

Shara, M. M., Moffat, A. F. J., Smith, L. F., et al. 1999, AJ, 118, 390

Smith, L., Shara, M., \& Moffat, A. 1996, MNRAS, 281, 163

Sota, A., Maíz Apellániz, J., Walborn, N. R., et al. 2011, ApJS, 193, 24

Sota, A., Maíz Apellániz, J., Morrell, N. I., et al. 2014, ApJS, 211, 10

Wilson, R. E., \& Devinney, E. J. 1971, ApJ, 166, 605

Page 6 is available in the electronic edition of the journal at http: //wWw . aanda. org 
Table 2. Observed heliocentric radial velocities of WR 68a.

\begin{tabular}{|c|c|c|c|c|c|c|c|}
\hline $\begin{array}{c}\text { HJD } \\
2450000+\end{array}$ & Phase & $\begin{array}{r}\text { N IV } \\
4057.76 \AA \\
{\left[\mathrm{km} \mathrm{s}^{-1}\right]} \\
\end{array}$ & $\begin{array}{r}\text { N III } \\
4640.64 \AA \\
{\left[\mathrm{km} \mathrm{s}^{-1}\right]} \\
\end{array}$ & $\begin{array}{r}\text { He II } \\
4685.68 \AA \\
{\left[\mathrm{km} \mathrm{s}^{-1}\right]} \\
\end{array}$ & $\begin{array}{r}\text { He II } \\
5411.52 \AA \\
{\left[\mathrm{km} \mathrm{s}^{-1}\right]} \\
\end{array}$ & $\begin{array}{r}\text { C IV } \\
5811.98 \AA \\
{\left[\mathrm{km} \mathrm{s}^{-1}\right]} \\
\end{array}$ & $\begin{array}{r}\text { Mean abs. } \\
\mathrm{H} \delta-\mathrm{H} \gamma-\mathrm{H} \beta \\
{\left[\mathrm{km} \mathrm{s}^{-1}\right]} \\
\end{array}$ \\
\hline 4189.83 & 0.99 & $-238 \pm 2$ & $-173 \pm 2$ & $58 \pm 1$ & $46 \pm 5$ & $-471 \pm 3$ & $-113 \pm 3$ \\
\hline 4189.85 & 0.99 & $-239 \pm 1$ & $-203 \pm 2$ & $41 \pm 2$ & $30 \pm 1$ & $-491 \pm 4$ & $-158 \pm 3$ \\
\hline 4191.86 & 0.38 & $54 \pm 2$ & $-85 \pm 2$ & $190 \pm 1$ & $274 \pm 2$ & $-68 \pm 5$ & $-290 \pm 6$ \\
\hline 4192.89 & 0.58 & & $-450 \pm 1$ & $-268 \pm 4$ & $-162 \pm 7$ & $-441 \pm 3$ & $-110 \pm 6$ \\
\hline 4193.67 & 0.72 & $-425 \pm 1$ & $-550 \pm 2$ & $-309 \pm 3$ & $-301 \pm 3$ & $-702 \pm 3$ & $10 \pm 9$ \\
\hline 4193.90 & 0.77 & $-483 \pm 2$ & $-542 \pm 1$ & $-297 \pm 1$ & $-327 \pm 4$ & $-742 \pm 3$ & $-15 \pm 9$ \\
\hline 4575.90 & 0.94 & $-297 \pm 1$ & $-281 \pm 2$ & $-64 \pm 1$ & $-113 \pm 3$ & $-571 \pm 3$ & $-36 \pm 3$ \\
\hline 4576.86 & 0.12 & $45 \pm 2$ & $-17 \pm 1$ & $256 \pm 1$ & $243 \pm 1$ & $-213 \pm 3$ & $-209 \pm 13$ \\
\hline 4577.75 & 0.29 & $150 \pm 2$ & $-38 \pm 2$ & $272 \pm 1$ & $340 \pm 1$ & $-32 \pm 5$ & $-283 \pm 17$ \\
\hline 4578.66 & 0.47 & & $-299 \pm 3$ & $6 \pm 1$ & $103 \pm 1$ & $-230 \pm 2$ & $-268 \pm 7$ \\
\hline 4578.89 & 0.51 & $-188 \pm 4$ & $-365 \pm 3$ & $-157 \pm 2$ & $-70 \pm 6$ & $-249 \pm 2$ & $-161 \pm 5$ \\
\hline 4579.61 & 0.65 & $-365 \pm 4$ & $-545 \pm 2$ & $-303 \pm 1$ & $-239 \pm 1$ & $-587 \pm 2$ & $-32 \pm 10$ \\
\hline 4579.88 & 0.70 & $-478 \pm 5$ & $-592 \pm 1$ & $-342 \pm 1$ & $-306 \pm 2$ & $-693 \pm 3$ & $-2 \pm 14$ \\
\hline 4633.69 & 0.01 & $-213 \pm 3$ & $-160 \pm 3$ & $66 \pm 3$ & $121 \pm 3$ & $-411 \pm 7$ & $-113 \pm 21$ \\
\hline 4914.87 & 0.87 & & $-415 \pm 3$ & $-177 \pm 3$ & $-270 \pm 2$ & & \\
\hline 4915.85 & 0.06 & & $18 \pm 1$ & $202 \pm 1$ & $231 \pm 3$ & & \\
\hline 4915.88 & 0.06 & & $-28 \pm 1$ & $214 \pm 1$ & $194 \pm 4$ & & \\
\hline 4916.81 & 0.24 & & $-8 \pm 3$ & $314 \pm 1$ & $308 \pm 1$ & & \\
\hline 4916.84 & 0.24 & & $33 \pm 4$ & $329 \pm 2$ & $326 \pm 1$ & & \\
\hline 4917.80 & 0.43 & $66 \pm 2$ & $-114 \pm 3$ & $120 \pm 1$ & $157 \pm 1$ & & $-304 \pm 13$ \\
\hline 4917.83 & 0.43 & $49 \pm 2$ & $-123 \pm 1$ & $111 \pm 2$ & $148 \pm 1$ & & $-283 \pm 12$ \\
\hline 5035.65 & 0.00 & $-256 \pm 2$ & $-128 \pm 2$ & $93 \pm 1$ & $66 \pm 2$ & & $-103 \pm 5$ \\
\hline 5038.62 & 0.57 & $-228 \pm 5$ & $-392 \pm 1$ & $-230 \pm 4$ & $-225 \pm 5$ & $-399 \pm 2$ & $-76 \pm 9$ \\
\hline 5039.64 & 0.77 & $-444 \pm 1$ & $-531 \pm 1$ & $-283 \pm 1$ & $-338 \pm 3$ & & $28 \pm 20$ \\
\hline 5059.56 & 0.58 & & $-440 \pm 3$ & $-194 \pm 3$ & $-131 \pm 3$ & & \\
\hline 5060.53 & 0.77 & & & $-304 \pm 2$ & $-296 \pm 2$ & & \\
\hline 5060.56 & 0.77 & & & $-317 \pm 1$ & $-234 \pm 1$ & & \\
\hline 5296.85 & 0.03 & & $-90 \pm 2$ & $135 \pm 2$ & & & \\
\hline 5297.81 & 0.22 & & & $317 \pm 1$ & $367 \pm 1$ & & \\
\hline 5410.52 & 0.81 & & $-557 \pm 2$ & $-239 \pm 1$ & & & \\
\hline 5411.55 & 0.00 & & $-158 \pm 2$ & $128 \pm 4$ & & & \\
\hline 5413.50 & 0.38 & & & $197 \pm 1$ & $258 \pm 1$ & & \\
\hline 5416.51 & 0.95 & & $-156 \pm 2$ & $-30 \pm 2$ & & & \\
\hline 5657.88 & 0.19 & & $73 \pm 2$ & $309 \pm 3$ & $268 \pm 4$ & & \\
\hline 5662.74 & 0.12 & & & $284 \pm 2$ & $216 \pm 1$ & & \\
\hline 6425.80 & 0.28 & & $2 \pm 3$ & $322 \pm 1$ & $353 \pm 3$ & & \\
\hline$\left.F W H M^{a}[\AA]\right]$ & & 14.6 & 30.0 & 22.5 & 21.6 & 34.5 & \\
\hline$E W^{a}[\AA ̊ \Omega$ & & -4.6 & -8.5 & -31.4 & -5.3 & -5.6 & \\
\hline
\end{tabular}

Notes. Radial velocities errors listed in table are provided by the fxcor task of IRAF. The errors are calculated from the asymmetric noise of the cross-correlation function and of the fitted height of the peak. ${ }^{(a)}$ The mean FWHM and EW of each emission line were measured in the disentangled spectra. 